\title{
Nota Científica \\ Produção de mudas de cedro em função de tipos de recipiente e fontes de fertilizante
}

\author{
Osmar Henrique de Castro Pias ${ }^{1 *}$, Juliano Berghetti ${ }^{1}$, Lucindo Somavilla ${ }^{1}$, Edson Bisognin Cantarelli ${ }^{1}$ \\ ${ }^{1}$ Universidade Federal de Santa Maria, Centro de Educação Superior Norte do RS, Linha Sete de Setembro, BR 386, km 40, CEP 98400000, Frederico Westphalen, \\ RS, Brasil
}

\author{
"Autor correspondente: \\ henriquepias@yahoo.com.br \\ Termos para indexação: \\ Cedrela fissilis \\ Meliaceae \\ Osmocote $\mathbb{R}$ \\ Kimcoat ${ }^{\circledR}$ \\ Nutrição \\ Index terms: \\ Cedrela fissilis \\ Meliaceae \\ Osmocote $\mathbb{R}$ \\ Kimcoat $($ ) \\ Nutrition
}

Histórico do artigo:

Recebido em 21/05/2014

Aprovado em 28/11/2014

Publicado em 30/06/2014

doi: 10.4336/2014.pfb.35.82.714
Resumo - O objetivo deste estudo foi avaliar a altura e o diâmetro do colo de mudas de cedro em função do tamanho de recipientes e fontes de adubação. Foram testados três tipos de recipiente (tubete, saco plástico e bombona) e três fontes de adubação (convencional, Kimcoat $($ e Osmocote $($ ) $)$ em sete avaliações. As mudas de cedro-rosa

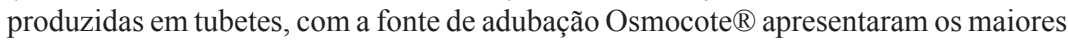
incrementos em altura e diâmetro do colo em comparação com as demais fontes de fertilizante. Os recipientes saco plástico e bombona proporcionaram crescimentos similares em altura das mudas. No entanto, as mudas produzidas em bombonas apresentaram maior crescimento em diâmetro do colo, em relação às plantadas em saco plástico.

\section{Produce of seedlings of cedar in function of types of container and fertilization sources}

\begin{abstract}
The aim of this study was to evaluate the production of cedar seedlings according to the size of containers and nutrient sources. It was tested three types of containers (Root trainers, plastic bag and plastic vase), three sources of fertilization (Conventional, Kimcoat ${ }^{\circledR}$ and Osmocote $($ ) in seven evaluations. The cedar seedlings in root trainers, fertilized with source Osmocote ${ }^{\circledR}$ presented the greatest increments in height and stem diameter when compared to another sources of fertilization. The plastic bag and plastic vase containers promoted similar seedlings height growth. However the seedlings grown in plastic vase presented greatest growth in stem diameter when compared with the ones in plastic bag.
\end{abstract}

Cedro-rosa (Cedrela fissilis (Vell.) Meliaceae) é uma espécie nativa do Brasil, com distribuição em praticamente todo o país (Lorenzi, 2002). A espécie apresenta grande importância econômica e ecológica, podendo ser empregada em marcenarias, construção naval e aeronáutica (Xavier et al., 2003). As mudas podem ser utilizadas em projetos paisagísticos, recuperação de áreas degradadas e reconstrução de matas ciliares (Lorenzi, 2002; Leles et al., 2006).
No processo de produção de mudas em viveiro, a escolha do recipiente adequado é fundamental, pois o tamanho apresenta influência direta na qualidade e no custo final das mudas (Barbosa et al., 2013). No Brasil, os recipientes mais recomendados atualmente são os tubetes de polietileno (menor volume) e os sacos plásticos, sendo que ambos exprimem vantagens e limitações (Ferraz \& Engel, 2011). Recipientes de maior tamanho proporcionam a redução do tempo de produção 
das mudas em viveiro e a obtenção de mudas de melhor qualidade (Brachtvogel \& Malavasi, 2010; Luca et al., 2010; Ferraz \& Engel, 2011; Caixeta et al., 2013). No entanto, apresentam como desvantagem a ocupação de maior espaço, volume de substrato, adubação, mão de obra, transporte e menor rendimento nos plantios (Ferraz \& Engel, 2011; Antoniazzi et al., 2013; Barbosa et al., 2013).

Outra importante técnica no processo de produção de mudas é o manejo das adubações. Visando aumentar a eficácia, tem-se adotado nos viveiros a utilização de fertilizantes de liberação controlada, devido à maior qualidade das mudas produzidas em comparação com fontes de fertilizantes convencionais (Yamanishi et al., 2004; Backes et al., 2007; Machado et al., 2011). O fertilizante de liberação controlada mais estudado e utilizado no Brasil para produção de mudas é o Osmocote ${ }^{\circledR}$. Recentemente outro fertilizante de liberação controlada que está sendo utilizado pelos produtores é o Kimcoat ${ }^{\circledR}$, no entanto, não há na literatura resultados que comprovem a sua eficiência na produção de mudas florestais.

O objetivo deste estudo foi avaliar a altura e o diâmetro do colo de mudas de cedro em função do tamanho de recipientes e fontes de adubação, considerando um período de até 90 dias após a repicagem.

$\mathrm{O}$ estudo foi conduzido em viveiro Florestal localizado em Frederico Westphalen $\left(27^{\circ} 23^{\prime} 82^{\prime}\right.$ 'S e $\left.53^{\circ} 25^{\prime} 35^{\prime} \mathrm{W}, 461 \mathrm{~m}\right)$. O delineamento experimental utilizado foi o de blocos casualizados em esquema trifatorial $(3 \times 3 \times 7)$, com três repetições de nove plantas por unidade experimental, conduzido em parcelas subdivididas. A parcela principal foi composta pelos tubetes de polietileno $\left(175 \mathrm{~cm}^{3}\right)$, sacos plásticos $(1.000$ $\left.\mathrm{cm}^{3}\right)$ e vasos de polietileno $\left(3.000 \mathrm{~cm}^{3}\right)$ (bombona). As subparcelas foram compostas pelas fontes de adubação Osmocote ${ }^{\circledR}$, Kimcoat ${ }^{\circledR}$ e convencional. O terceiro fator analisado foi a época de avaliação, que foram de $0 ; 15$; 30, 45; 60; 75 e 90 dias após a repicagem (DAR).

Para o cálculo da quantidade de fertilizantes de todos os tratamentos, tomou-se como base a recomendação do fabricante da fonte Osmocote ${ }^{\circledR}$ (15-09-12), que foi de $6 \mathrm{~kg} \mathrm{~m}^{-3}$ de substrato. O Kimcoat $\AA$ é comercializado com a tecnologia de liberação controlada em grânulos de ureia (45\% de N), MAP (14-34-00) e cloreto de potássio (60\%), sendo aplicados 1,51, 1,59 e $1,2 \mathrm{~kg} \mathrm{~m}^{-3}$ de substrato, respectivamente. A fonte convencional constituiu-se de ureia $(45 \%$ de $\mathrm{N})$, superfosfato triplo ( $46 \%$ de $\mathrm{P})$ e cloreto de potássio (60\% de K), aplicados nas quantidades de 2, 1,17 e $1,2 \mathrm{~kg} \mathrm{~m}-3$ de substrato, respectivamente. O substrato utilizado em todos os recipientes foi constituído de 50\% de solo argiloso (horizonte B de Latossolo Vermelho distrófico), $30 \%$ de vermiculita e $20 \%$ de areia média lavada. Os fertilizantes de liberação controlada e o P do tratamento convencional foram incorporados ao substrato de cada tratamento, na hora da repicagem. $\mathrm{O}$ $\mathrm{N}$ e o K do tratamento convencional foram parcelados com aplicações de cobertura a cada 30 dias.

As mudas foram produzidas em canteiro de raiz nua, contendo substrato estéril como meio de cultivo, sob o regime de casa de vegetação e irrigação automatizada. Aos 40 dias após a semeadura, foi realizada a repicagem das mudas de maior vigor e uniformes para as embalagens definitivas (altura média de $5,78 \mathrm{~cm}$ ), em substrato com os respectivos fertilizantes. As avaliações das mudas constituíram-se das medidas de altura $(\mathrm{H})$ e diâmetro do colo (DC). A H foi mensurada com régua graduada, tomando-se como base a medida do colo da planta até o meristema apical e o DC medido com paquímetro digital.

Os resultados obtidos foram inicialmente submetidos à análise de variância pelo teste $\mathrm{F}(\mathrm{p} \leq 0,05) \mathrm{Na}$ sequência, as médias qualitativas foram comparadas pelo teste de Tukey $(\mathrm{p} \leq 0,05)$, e as médias quantitativas pela regressão polinomial, através do programa Statistical Analysis System - SAS 9.3 (SAS Inc, Cary, EUA).

A partir do teste de variância, constatou-se que não houve interação tripla entre os fatores de estudo. Contudo, ocorreram interações duplas para ambas as variáveis ( $\mathrm{H} \mathrm{e} \mathrm{DC)} \mathrm{entre} \mathrm{as} \mathrm{épocas} \mathrm{de} \mathrm{avaliação} \mathrm{e} \mathrm{os} \mathrm{tipos}$ de recipientes, fontes de adubação e tipos de recipientes e, épocas de avaliação e fontes de adubação.

Independente do tipo de recipiente ou fonte de adubação (Figuras 1a e 1b) ocorreu incremento linear da altura das mudas com o aumento do tempo de permanência das mesmas no viveiro. As mudas produzidas em sacos plásticos e bombonas apresentaram crescimentos maiores em comparação com as produzidas em tubetes. Ferraz \& Engel (2011) citam que a utilização de recipientes maiores reduz o tempo de permanência das mudas no viveiro. Neste caso, as mudas produzidas nos maiores recipientes atingiram o tamanho das produzidas em tubetes, com 60 dias de antecedência, no entanto, para que essas mudas fossem levadas para o campo prematuramente seria necessário uma avaliação da viabilidade do sistema radicular das mesmas. 
Até os $30 \mathrm{DAR}$ as mudas produzidas nas diferentes fontes de adubação não apresentaram diferenças em $\mathrm{H}$ (Figura 1c). No entanto, a partir dos 45 DAR as mudas adubadas com o Osmocote ${ }^{\circledR}$ apresentaram maior crescimento em altura que as adubadas com as outras duas fontes de fertilizante, corroborando com os resultados de Yamanishi et al. (2004) e Machado et al. (2011) em que observaram superioridade de
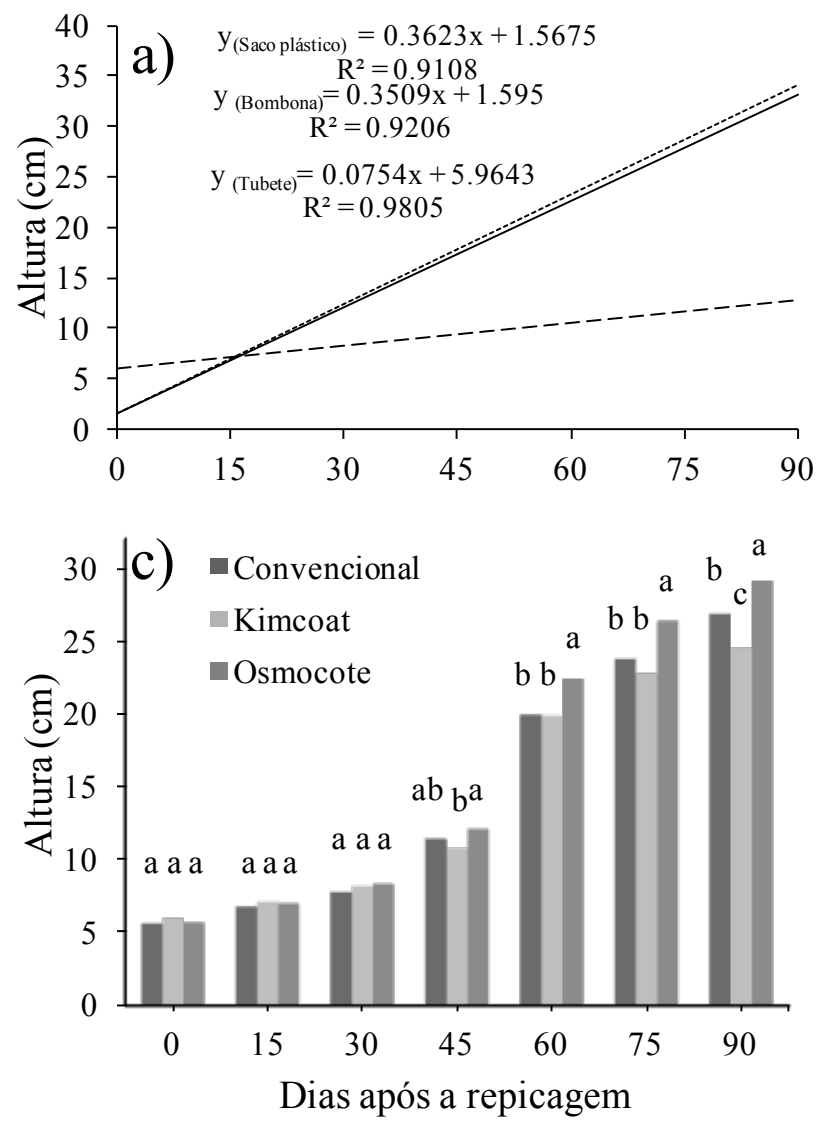

crescimento das mudas produzidas com Osmocote $\AA$. As mudas produzidas com Kimcoat ${ }^{\circledR}$ não apresentaram diferenças das mudas produzidas com fertilizante convencional, com exceção da avaliação aos 90 DAR (última avaliação) em que observou-se desenvolvimento inferior.
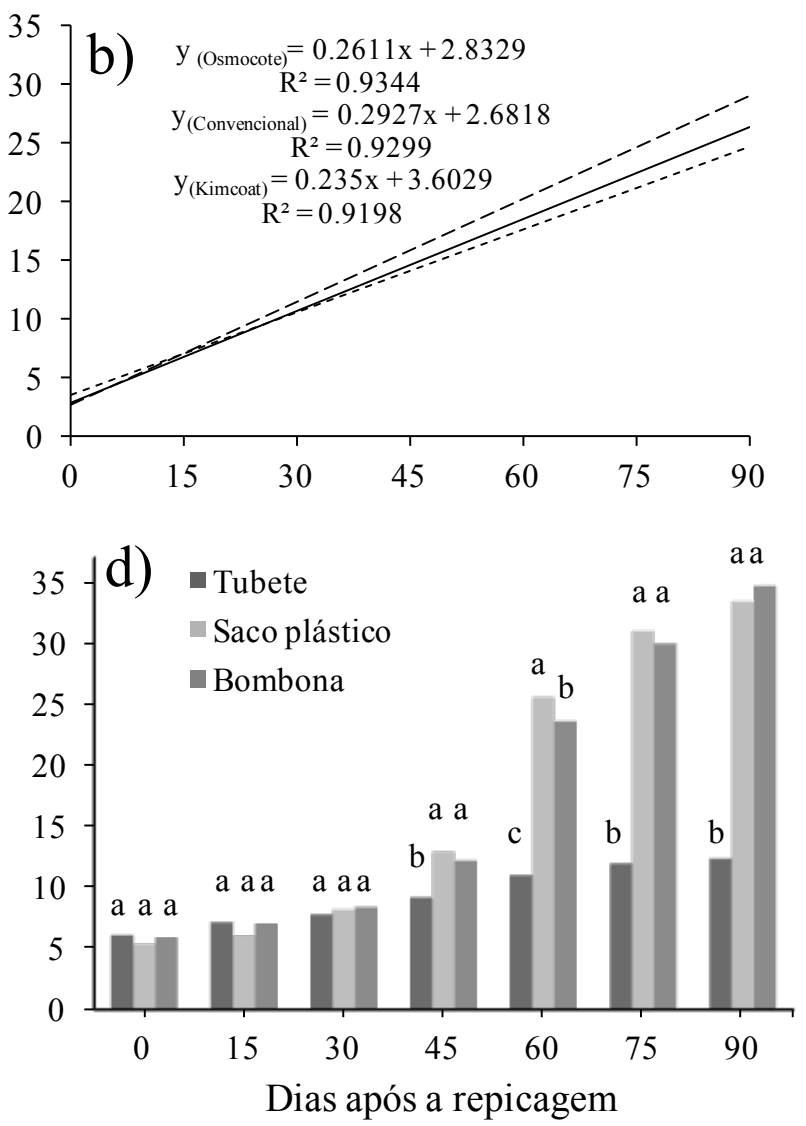

Figura 1. Altura das mudas de cedro em função: a) época de avaliação em cada tipo de recipiente. b) época de avaliação em cada fonte de adubação. c) fonte de adubação em cada época de avaliação. d) tipo de recipiente em cada época de avaliação, em Frederico Westphalen, RS, 2012/2013. * Tratamento com letras iguais não diferem dentro de cada fonte de variação, pelo teste de Tukey a $5 \%$ de probabilidade.

As mudas produzidas nos diferentes tipos de recipiente apresentaram diferenças em $\mathrm{H}$ apenas a partir dos 45 DAR (Figura 1d), contrastando-se aos resultados descritos por Barbosa et al. (2013), em que observaram diferenças dos incrementos em $\mathrm{H}$ de mudas florestais nativas produzidas em diferentes recipientes apenas nos primeiros 30 DAR. A partir dos $45 \mathrm{DAR}$, em todas as demais avaliações, as mudas dos recipientes de maior tamanho (bombona e saco plástico) tiveram maior crescimento que as transplantadas nos tubetes (Figura 2a). Resultados similares são reportados por Luca et al. (2010) e Ferraz \& Engel (2011), onde os maiores recipientes proporcionaram maior desenvolvimento em $\mathrm{H}$ das mudas.

Nas mudas produzidas em bombona e saco plástico, ocorreram diferenças significativas apenas na avaliação aos 60 DAR, em que as mudas em saco plástico foram maiores. Nas demais avaliações os valores não diferiram estatisticamente. 
As mudas produzidas com Osmocote ${ }^{\circledR}$ e em sacos plásticos obtiveram maiores incrementos do que as produzidas em bombonas. Este resultado provavelmente está relacionado às diferentes dimensões dos recipientes, sendo que o saco plástico, apesar de seu menor volume total, apresenta dimensão mais estreita e comprida que as bombonas, podendo este fator ter favorecido o desenvolvimento radicular das mudas e consequentemente a $\mathrm{H}$.

A altura das mudas produzidas em tubetes com o fertilizante Osmocote ${ }^{\circledR}$, apresentaram as maiores médias de $\mathrm{H}$, demonstrando a eficiência deste fertilizante.
As mudas produzidas em sacos plásticos com adição de Osmocote ${ }^{\circledR}$, não se diferenciaram do fertilizante convencional, e este do Kimcoat ${ }^{\circledR}$. No recipiente bombona as fontes de adubação não apresentaram diferenças. A semelhança de crescimento em $\mathrm{H}$ das mudas submetidas a diferentes fontes de adubação nos maiores recipientes está relacionada, provavelmente, ao maior volume de substrato e consequentemente maior disponibilidade de fertilizante, já que os fertilizantes foram adicionados proporcionalmente ao volume de substrato, assim como a disponibilidade de maior espaço para o crescimento das raízes.

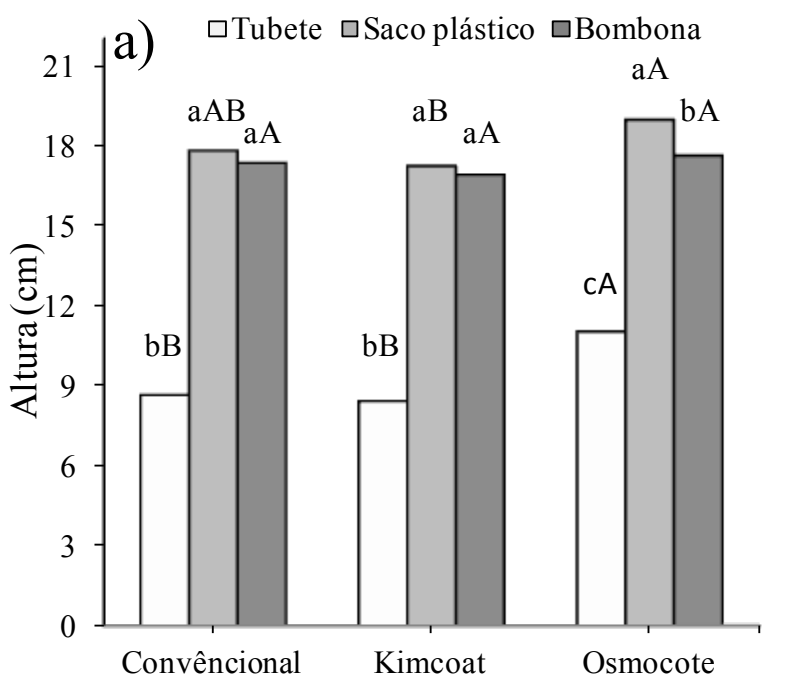

Fontes de adubação b) $\square$ Convêncional $\square$ Kimcoat $\square$ Osmocote

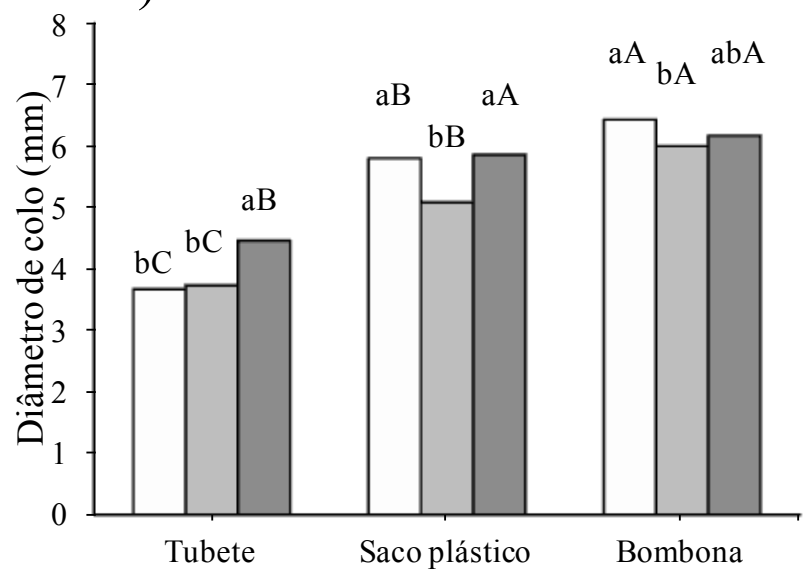

Tipos de recipiente

Figura 2. Desdobramento da interação entre tipos de recipiente e fontes de adubação para as variáveis: a) altura média das mudas de cedro; b) diâmetro médio de colo das mudas de cedro, em Frederico Westphalen, RS, $2012 / 2013$. *Tratamento com letras iguais minúsculas não diferem dentro de cada fonte de adubação, letras maiúsculas iguais não diferem entre os recipientes, pelo teste de Tukey a 5\% de probabilidade.

Considerando as fontes de adubação convencional e Kimcoat ${ }^{\circledR}$, as dimensões dos DC das mudas de cedro (Figura 2b) foram mais elevadas nos recipientes maiores. $\mathrm{Na}$ fonte de adubação Osmocote ${ }^{\circledR}$, os recipientes sacos plásticos e bombonas não apresentaram diferenças, porém foram maiores que as mudas nos tubetes. Luca et al. (2010), testando diferentes tipos de recipientes para a mesma espécie, observaram comportamento das mudas semelhante ao deste estudo. Esses autores citam a importância de se obter uma muda com crescimento equilibrado entre o desenvolvimento em $\mathrm{H}$ e DC. O DC nas mudas com Osmocote ${ }^{\circledR}$ foi superior às demais fontes, contudo, no recipiente bombona não ocorreu diferença do Osmocote ${ }^{\circledR}$ e o fertilizante convencional.

Independente do recipiente e da fonte de adubação, houve aumento linear do diâmetro das mudas com o aumento do tempo de permanência no viveiro, contudo, as mudas produzidas em bombonas (Figura 3a) e as adubadas com Osmocote ${ }^{\circledR}$ (Figura 3b) apresentaram os maiores incrementos. 

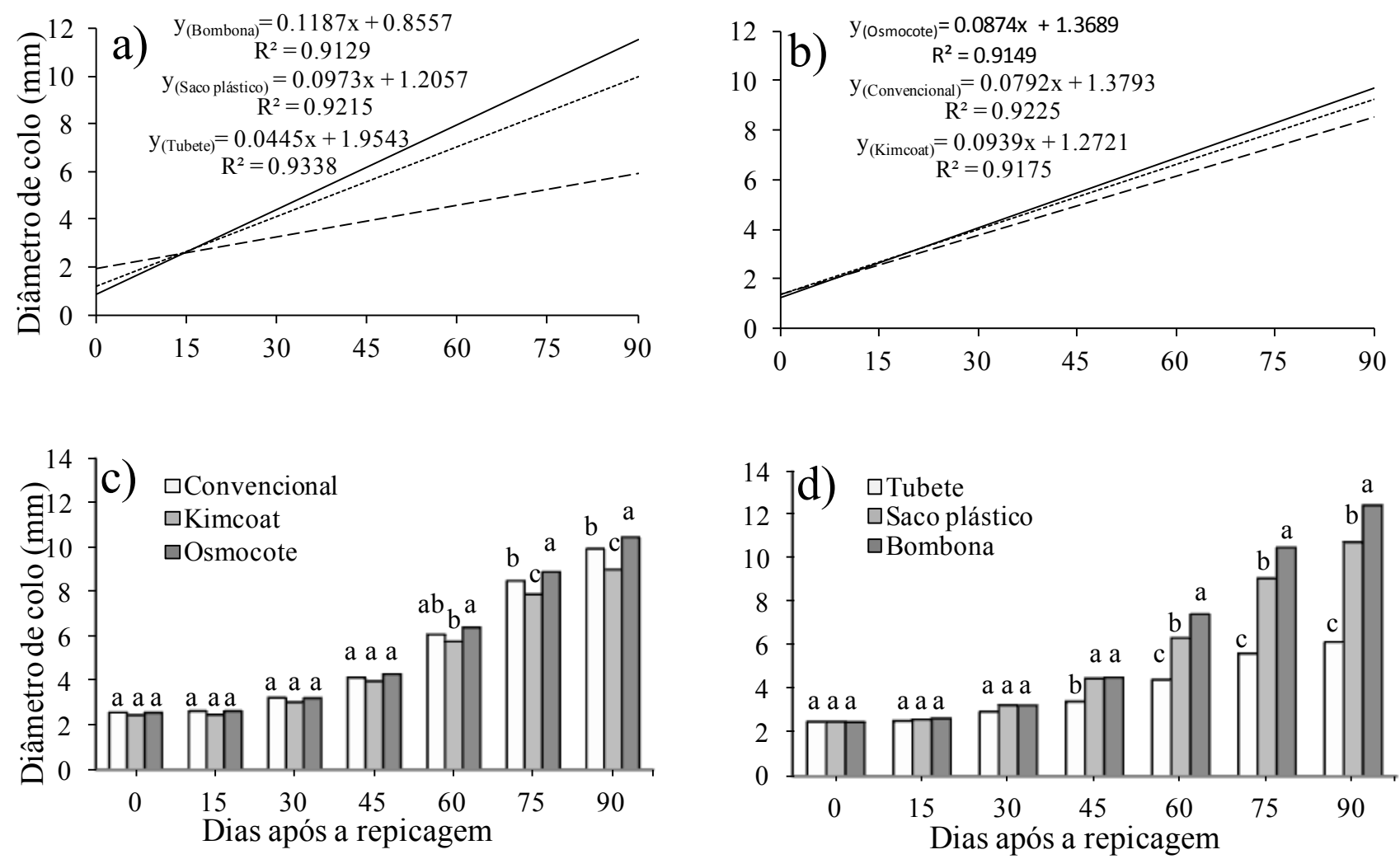

Figura 3. Diâmetro de colo das mudas de cedro em função: a) época de avaliação dentro de cada tipo de recipiente. b) época de avaliação dentro de cada fonte de adubação. c) fontes de adubação dentro de cada época de avaliação. d) Tipo de recipiente dentro de cada época de avaliação, em Frederico Westphalen, RS, 2012/2013. *Tratamentos seguidos de mesma letra não diferem significativamente pelo teste de Tukey a $5 \%$ de probabilidade.

Houve diferença estatística do DC das mudas quando submetidas a diferentes fontes de adubação apenas após os 60 DAR (Figura 3c), sendo que o Osmocote ${ }^{\circledR}$ foi a que proporcionou o melhor resultado. Os menores incrementos de DC das mudas foram obtidos com a aplicação de Kimcoat. O DC das mudas em função dos tipos de recipiente apresentou diferenças aos 45 DAR, sendo que a bombona e o saco plástico foram superiores. Nas avaliações subsequentes as mudas produzidas nas bombonas foram as que apresentaram os maiores incrementos, seguidos pelas produzidas em sacos plásticos.

\section{Conclusões}

As mudas de cedro-rosa produzidas em tubetes, com a fonte de adubação Osmocote ${ }^{\circledR}$ apresentaram os maiores incrementos em altura e diâmetro do colo em comparação com as demais fontes de fertilizante.
Os recipientes saco plástico e bombona proporcionam crescimentos similares em altura das mudas de cedrorosa. No entanto, as mudas produzidas em bombonas apresentaram maior crescimento em diâmetro do colo, em relação às plantadas em saco plástico.

\section{Referências}

ANTONIAZZI, A. P.; BINOTTO, B.; NEUMANN, G. M.; SAUSEN, T. L.; BUDKE, J. C. Eficiência de recipientes no desenvolvimento de mudas de Cedrela fissilis Vell. (Meliaceae). Revista Brasileira de Biociências, Porto Alegre, RS, v. 11, n. 3, p. 313-317, 2013.

BACKES, C.; FERNANDES, F. M.; KROHN, N. G.; LIMA, C.P.; KIIHL, T. A. M. Produção de pimenta ornamental em função de substratos e doses de adubação com fertilizantes de liberação lenta e tradicional. Scientia Agraria Paranaensis, Marechal Candido Randon, v. 6, p. 67-76, 2007.

BARBOSA, T. C.; RODRIGUES, R. B.; COUTO, H. T. Z. Tamanhos de recipientes e o uso de hidrogel no estabelecimento de mudas de espécies florestais nativas. Hoehnea, São Paulo, v. 40, n. 3, p. 537-556, 2013. 
BRACHTVOGEL, E. L.; MALAVASI, U. C. Volume do recipiente, adubação e sua forma de mistura ao substrato no crescimento inicial de Peltophorum dubium (Sprengel) taubert em viveiro. Revista Árvore, Viçosa, MG, v. 34, n. 2, p. 223-232, 2010.

CAIXETA, A. F. B.; REIS, J. M. R.; RODRIGUES, J. F. Produção de mudas de jatobá em diferentes dimensões de recipientes e composições de substratos. Revista Agrotecnologia, Anápolis, v. 4, n. 1, p. 46-57, 2013.

FERRAZ, A. V.; ENGEL, V. L. Efeito do tamanho de tubetes na qualidade de mudas de jatobá (Hymenaea courbaril L. var. Stilbocarpa (Hayne) Lee et Lang.), ipê-amarelo (Tabebuia chrysotricha (Mart. Ex DC.) Sandl.) e guarucaia (Parapiptadenia rigida (Benth.) Brenan). Revista Árvore, Viçosa, $\mathrm{MG}$, v. 35, n. 3, p. 413-423, 2011.

LELES, P. S. S.; LISBOA, A. C.; NETO, S. N. O.; GRUGIKI, M. A.; FERREIRA, M. A. Qualidade de mudas de quatro espécies florestais produzidas em tubetes de diferentes dimensões. Floresta e Ambiente, Rio de Janeiro, v. 13, n. 1, p. 69-78, 2006.
LORENZI, H. Árvores brasileiras: manual de identificação e cultivo de plantas arbóreas do Brasil. 4. ed. Odessa: Instituto Plantarum, 2002. v. 1.158 p.

LUCA, E. F.; REBECCHI, R. J.; SCHORN, L. A. Crescimento e qualidade de mudas de cedro (Cedrela fissilis Vellozo) em viveiro, mediante diferentes técnicas de produção. Revista do Instituto Florestal, São Paulo, v. 22, n. 2, p. 189-199, 2010.

MACHADO, D. L. M.; LUCENA, C. C.; SANTOS, D.; SIQUEIRA, D. L.; MATARAZZO, P. H. M.; STRUIVIMG, T. B. Slow-release and organic fertilizers on early growth of Rangpur lime. Revista Ceres, Viçosa, MG, v. 58, n. 3, p. 359-365, 2011.

XAVIER, A.; SANTOS, G. A.; OLIVEIRA, M. L. Enraizamento de miniestaca caulinar e foliar na propagação vegetativa de cedro-rosa (Cedrela fissilis Vell.). Revista Árvore, Viçosa, MG, v. 27, n. 3, p. 351-356, 2003.

YAMANISHI, O. K.; FAGUNDES, G. R.; MACHADO FILHO, J. A.; VALONE, G. V. Efeito de diferentes substratos e duas formas de adubação na produção de mudas de mamoeiro. Revista Brasileira de Fruticultura, Jaboticabal, v. 26, n. 2, p. 276-279, 2004. 